\title{
Supporting Information: Substrate Orientation in 4-Oxalocrotonate Tautomerase and its Effect on QM/MM Energy Profiles.
}

\author{
Tell Tuttle ${ }^{*}$ and Walter Thiel ${ }^{\dagger}$
}

Max-Planck-Institut für Kohlenforschung, D-45470 Mülheim an der Ruhr, Germany,

Email: thiel@mpi-muelheim.mpg.de, tell@mpi-muelheim.mpg.de

1. QM/MM energy decomposition analysis.

2. Extended basis set calculations.

3. QM/MM single point calculations with different density functionals.

4. Basis set specification for def2-TZVP+d.

5. PDB coordinates for $\mathrm{QM} / \mathrm{MM}$ optimized structures. 


\section{QM/MM energy decomposition analysis.}

The QM/MM energy decomposition terms are described in the main text. The individual contributions of each term described in eqs. (1) and (2) are given in Tables S1S3, for models A-C, respectively. $E_{Q M / M M-q}$ is the energy contribution from the

interaction of the $\mathrm{QM}$ region with the charges in the $\mathrm{MM}$ region. $\Delta E_{\mathrm{QM} / M M-o}$ is the energy contribution from the other interactions between the QM and $\mathrm{MM}$ regions. These include the van der Waals interactions, as well as the bonding MM contributions that occur across the QM/MM boundary. 
Table S1. Decomposition of the total energy for the calculated reaction path in model A. Absolute energies are in hartree. Relative energies in $\mathrm{kcal} / \mathrm{mol}$, relative to the reactant.

\begin{tabular}{ccccccccc}
\hline Structure & $E_{\text {Total }}$ & $E_{(Q M, M M)}$ & $E_{(M M, Q M)}$ & $E_{Q M}$ & $E_{Q M / M M-q}$ & $E_{M M}$ & $E_{Q M / M M-o}$ & $E_{Q M / M M}$ \\
\hline \hline React & -953.36350 & -933.20457 & -20.15893 & -933.17804 & -0.02653 & -20.12986 & -0.02907 & -0.05560 \\
TS1 & -953.31715 & -933.17536 & -20.14179 & -933.09467 & -0.08069 & -20.11578 & -0.02602 & -0.10670 \\
INT & -953.32133 & -933.18492 & -20.13641 & -933.08564 & -0.09928 & -20.11406 & -0.02235 & -0.12163 \\
TS2 & -953.31643 & -933.17517 & -20.14126 & -933.10349 & -0.07168 & -20.11871 & -0.02255 & -0.09423 \\
Prod & -953.37753 & -933.21827 & -20.15926 & -933.18300 & -0.03527 & -20.13213 & -0.02713 & -0.06240 \\
\hline & & & & & & & & \\
\hline Structure & $\Delta E_{\text {Total }}$ & $\Delta E_{(Q M, M M)}$ & $\Delta E_{(M M, Q M)}$ & $\Delta E_{Q M}$ & $\Delta E_{Q M / M M-q}$ & $\Delta E_{M M}$ & $\Delta E_{Q M / M M-o}$ & $\Delta E_{Q M / M M}$ \\
\hline \hline React & 0.00 & 0.00 & 0.00 & 0.00 & 0.00 & 0.00 & 0.00 & 0.00 \\
TS1 & 29.09 & 18.33 & 10.76 & 52.32 & -33.98 & 8.84 & 1.92 & -32.07 \\
INT & 26.46 & 12.33 & 14.13 & 57.98 & -45.65 & 9.92 & 4.21 & -41.44 \\
TS2 & 29.54 & 18.45 & 11.09 & 46.78 & -28.33 & 7.00 & 4.09 & -24.24 \\
Prod & -8.80 & -8.59 & -0.21 & -3.11 & -5.48 & -1.42 & 1.22 & -4.27 \\
\hline
\end{tabular}


Table S2. Decomposition of the total energy for the calculated reaction path in model B. Absolute energies are in hartree. Relative energies in $\mathrm{kcal} / \mathrm{mol}$, relative to the reactant.

\begin{tabular}{ccccccccc}
\hline Structure & $E_{\text {Total }}$ & $E_{(Q M, M M)}$ & $E_{(M M, Q M)}$ & $E_{Q M}$ & $E_{Q M / M M-q}$ & $E_{M M}$ & $E_{Q M / M M-o}$ & $E_{Q M / M M}$ \\
\hline \hline React & -999.34836 & -933.83316 & -65.51521 & -933.16938 & -0.66378 & -65.50344 & -0.01176 & -0.67554 \\
TS1 & -999.32774 & -933.83269 & -65.49505 & -933.11834 & -0.71435 & -65.49329 & -0.00176 & -0.71611 \\
INT & -999.33135 & -933.85033 & -65.48102 & -933.09664 & -0.75370 & -65.48283 & 0.00181 & -0.75188 \\
TS2 & -999.31902 & -933.82843 & -65.49058 & -933.11200 & -0.71643 & -65.48434 & -0.00624 & -0.72267 \\
Prod & -999.34526 & -933.83656 & -65.50870 & -933.16674 & -0.66982 & -65.49789 & -0.01081 & -0.68063 \\
\hline
\end{tabular}

\begin{tabular}{ccccccccc}
\hline Structure & $\Delta E_{\text {Total }}$ & $\Delta E_{(Q M, M M)}$ & $\Delta E_{(M M, Q M)}$ & $\Delta E_{Q M}$ & $\Delta E_{Q M / M M-q}$ & $\Delta E_{M M}$ & $\Delta E_{Q M / M M-o}$ & $\Delta E_{Q M / M M}$ \\
\hline \hline React & 0.00 & 0.00 & 0.00 & 0.00 & 0.00 & 0.00 & 0.00 & 0.00 \\
TS1 & 12.94 & 0.29 & 12.65 & 32.03 & -31.73 & 6.37 & 6.28 & -25.46 \\
INT & 10.68 & -10.78 & 21.45 & 45.65 & -56.42 & 12.93 & 8.52 & -47.90 \\
TS2 & 18.41 & 2.96 & 15.45 & 36.00 & -33.04 & 11.99 & 3.47 & -29.57 \\
Prod & 1.95 & -2.13 & 4.08 & 1.65 & -3.79 & 3.49 & 0.60 & -3.19 \\
\hline
\end{tabular}


Table S3. Decomposition of the total energy for the calculated reaction path in model C. Absolute energies are in hartree. Relative energies in $\mathrm{kcal} / \mathrm{mol}$, relative to the reactant. The “_- “ indicate that the SCF calculation in the gas phase did not converge.

\begin{tabular}{ccccccccc}
\hline Structure & $E_{\text {Total }}$ & $E_{(Q M, M M)}$ & $E_{(M M, Q M)}$ & $E_{Q M}$ & $E_{Q M / M M-q}$ & $E_{M M}$ & $E_{Q M / M M-o}$ & $E_{Q M / M M}$ \\
\hline \hline React & -951.55735 & -933.60438 & -17.95297 & -933.20896 & -0.39542 & -17.93023 & -0.02274 & -0.41816 \\
TS1 & -951.52753 & -933.60003 & -17.92751 & - & - & -17.90807 & -0.01944 & - \\
INT & -951.54611 & -933.64120 & -17.90492 & - & - & -17.89335 & -0.01156 & - \\
TS2 & -951.52745 & -933.59198 & -17.93547 & - & - & -17.91953 & -0.01594 & - \\
Prod & -951.57261 & -933.60669 & -17.96592 & -933.22068 & -0.38601 & -17.94665 & -0.01927 & -0.40528 \\
\hline Structure & $\Delta E_{\text {Total }}$ & $\Delta E_{(Q M, M M)}$ & $\Delta E_{(M M, Q M)}$ & $\Delta E_{Q M}$ & $\Delta E_{Q M / M M-q}$ & $\Delta E_{M M}$ & $\Delta E_{Q M / M M-o}$ & $\Delta E_{Q M / M M}$ \\
\hline \hline React & 0.00 & 0.00 & 0.00 & 0.00 & 0.00 & 0.00 & 0.00 & 0.00 \\
TS1 & 18.71 & 2.73 & 15.98 & - & - & 13.91 & 2.07 & - \\
INT & 7.05 & -23.10 & 30.15 & - & - & 23.14 & 7.01 & - \\
TS2 & 18.76 & 7.78 & 10.98 & - & - & 6.71 & 4.26 & - \\
Prod & -9.58 & -1.45 & -8.13 & -7.36 & 5.91 & -10.30 & 2.17 & 8.08 \\
\hline
\end{tabular}




\section{Extended basis set calculations.}

Results from the BLYP/def2-TZVP+d single-point calculations of the model A and model B structures. The QM region includes only the substrate and P1.

Table S4. Relative energies from single-point QM/MM calculations with larger basis set. Energies in $\mathrm{kcal} / \mathrm{mol}$, relative to the reactant.

\begin{tabular}{|c|c|c|c|c|}
\hline System & $\Delta E_{\text {Total }}$ & $\Delta E_{(Q M, M M)}$ & $\Delta E_{(M M, Q M)}$ & $\Delta E_{Q M}$ \\
\hline \multicolumn{5}{|c|}{ Model A } \\
\hline React & 0.00 & 0.00 & 0.00 & 0.00 \\
\hline TS1 & 28.07 & 17.31 & 10.76 & 41.49 \\
\hline INT & 25.06 & 10.93 & 14.13 & 66.65 \\
\hline TS2 & 28.38 & 17.29 & 11.09 & 39.00 \\
\hline Prod & -8.98 & -8.77 & -0.21 & -3.91 \\
\hline \multicolumn{5}{|c|}{ Model B } \\
\hline React & 0.00 & 0.00 & 0.00 & 0.00 \\
\hline TS1 & 12.41 & -0.24 & 12.65 & 25.38 \\
\hline INT & 9.98 & -11.47 & 21.45 & 31.62 \\
\hline TS2 & 17.89 & 2.44 & 15.45 & 28.66 \\
\hline Prod & 1.98 & -2.10 & 4.08 & 0.32 \\
\hline
\end{tabular}




\section{QM/MM single point calculations with different density functionals.}

In order to test the functional dependence of the energy profile calculated for model $\mathrm{C}$ we have performed single point calculations on the BLYP/TZVPP/CHARMM optimized structures. The resulting $\mathrm{QM} / \mathrm{MM}$ energies show variations around $5 \mathrm{kcal} / \mathrm{mol}$, where the BLYP energies reported in the main paper are in the middle of this range.

Table S5. Single point QM/MM calculations on BLYP/TZVPP/CHARMM optimized structures with four different functionals. ${ }^{\mathrm{a}}$

\begin{tabular}{llllll}
\hline & BLYP & BP86 & B3LYP & TPSS & TPSSH \\
\hline TS1 & 18.7 & 15.4 & 21.7 & 17.8 & 19.4 \\
INT & 7.0 & 5.6 & 8.3 & 6.3 & 7.2 \\
TS2 & 18.8 & 15.4 & 21.1 & 17.6 & 19.1 \\
PROD & -9.6 & -9.9 & -9.9 & -10.3 & -10.3
\end{tabular}

\footnotetext{
${ }^{a}$ All calculations employ CHARMM as the MM method and the TZVPP basis set for the QM region. Energies are given relative to the reactant state and are reported in $\mathrm{kcal} / \mathrm{mol}$.
} 


\section{Basis set specification for def2-TZVP+d.}

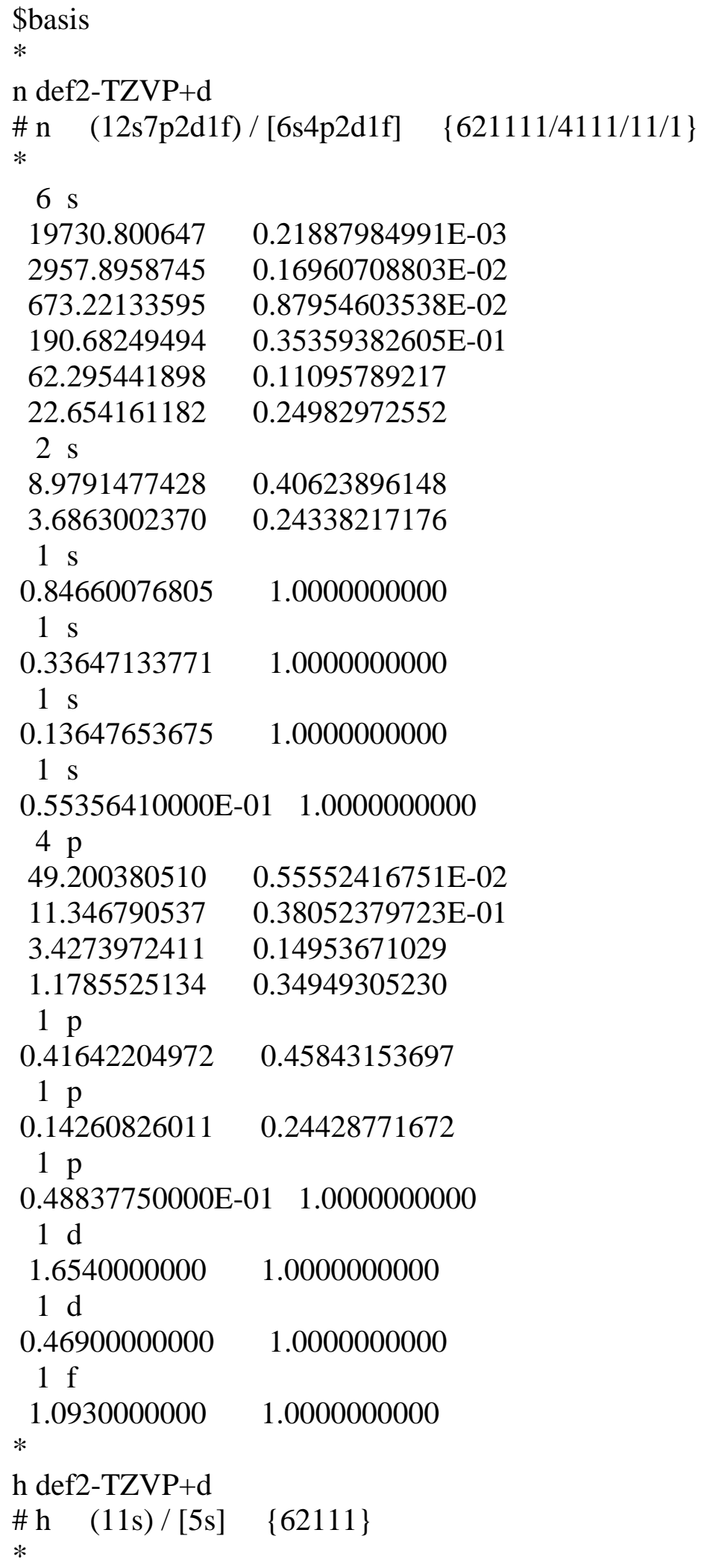




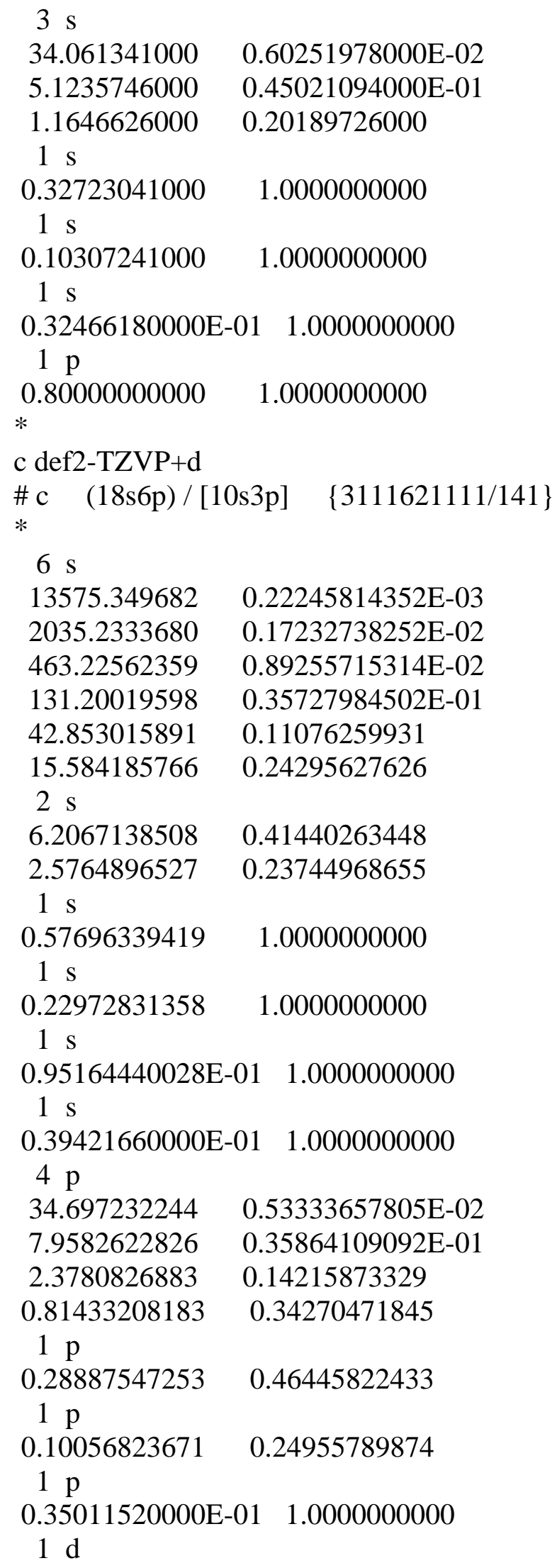




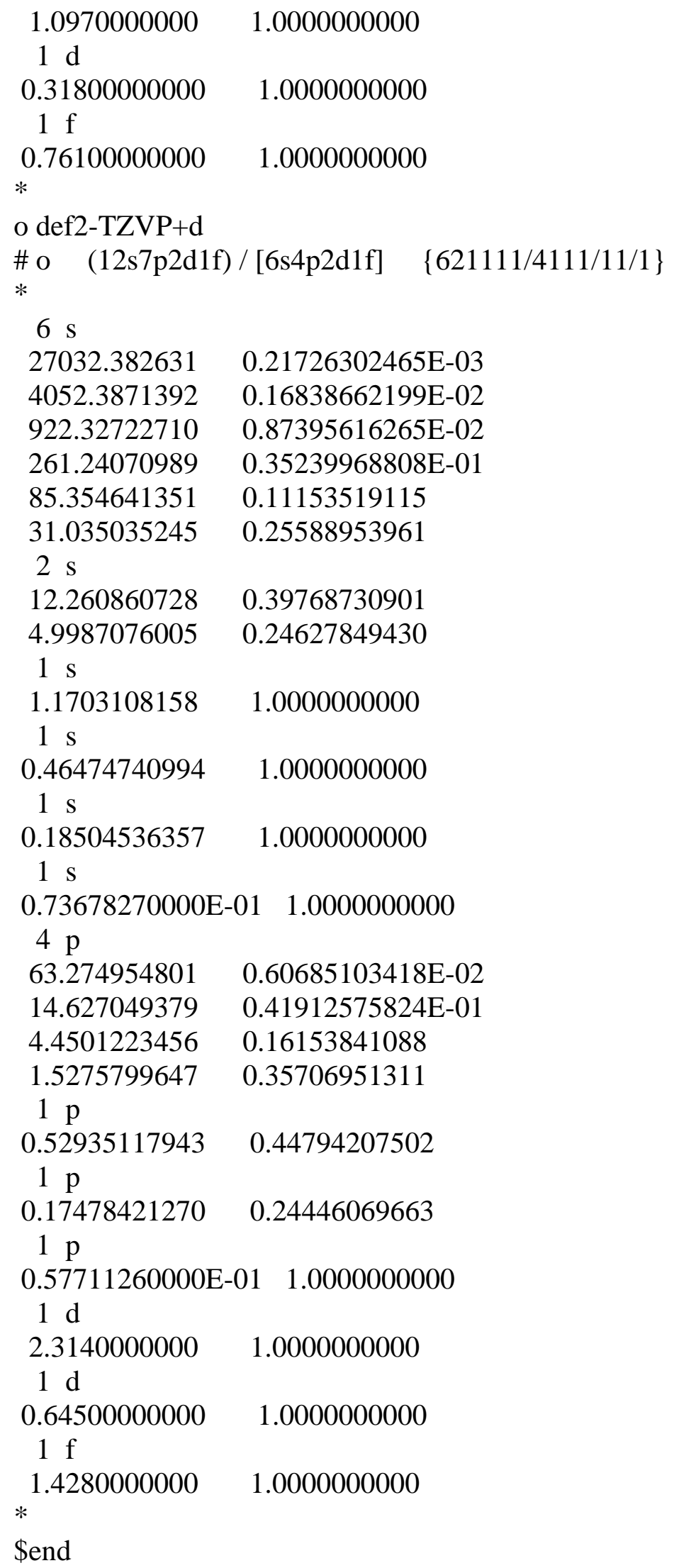

Send 


\section{PDB coordinates for QM/MM optimized structures.}

The PDB coordinates (in a separate PDB file) correspond to the final QM/MM (BLYP/CHARMM) optimized geometry of the model A and model C structures discussed in the main text. 